\title{
WEAK RESTRICTED AND VERY RESTRICTED OPERATORS ON $L^{2}$
}

BY

\author{
J. MARSHALL ASH
}

\author{
Dedicated to the memory of Karel de Leeuw
}

\begin{abstract}
A battlement is a real function with values in $\{0,1\}$ that looks like a castle battlement. A commuting with translation linear operator $T$ mapping step functions on $\mathbf{R}$ into the set of all measurable functions on $\mathbf{R}$ and satisfying $\|T b\|_{2} \leqslant C\|b\|_{2}$ for all battlements $b$ is bounded on $L^{2}(\mathbf{R})$. This remains true if the underlying space is the circle but is demonstrably false if the underlying space is the integers. Michael Cowling's theorem that linear commuting with translation operators are bounded on $L^{2}$ if they are weak restricted $(2,2)$ is reproved and an application of this result to sums of exponentials is given.
\end{abstract}

1. Introduction. The group $G$ will be either $\mathbf{R}$ - the real numbers, $\mathbf{T}=[0,1]$ - the torus, or $\mathbf{Z}$-the integers; each endowed with its usual measure. All sets and functions will be Lebesgue measurable. All operators will be linear. By $L^{2}(G)$ we mean functions having $\|f\|_{2}=\left(\int_{G}|f|^{2}\right)^{1 / 2}$ finite when $G=\mathbf{R}$ or $\mathbf{T}$ and $\|f\|_{2}=$ $\left(\Sigma|f(n)|^{2}\right)^{1 / 2}$ finite when $G=\mathbf{Z}$. Say that a linear operator $T$ is of strong type $(2,2)$, and write $T \in s .(2,2)$ if there is a constant $C$, such that for every $f \in L^{2}(G)$, $T f \in L^{2}(G)$ and

$$
\|T f\|_{2} \leqslant C\|f\|_{2} .
$$

Say that $T$ is of restricted type $(2,2)$ and write $T \in r .(2,2)$ if $(1.1)$ holds whenever $f$ is the characteristic function of a measurable set.

Tchebyshev's theorem states that

$$
\sup _{\alpha>0} \operatorname{meas}\left\{x \in G:|g|^{2}>\alpha\right\} \cdot \alpha \leqslant \int_{G}|g|^{2},
$$

so defining $\|g\|_{2, \infty}^{*}$ to be the square root of the left hand-side of (1.2) we have

$$
\|g\|_{2, \infty}^{*} \leqslant\|g\|_{2},
$$

which together with (1.1) implies that strong type $(2,2)$ operators $T$ also satisfy

$$
\|T f\|_{2, \infty}^{*} \leqslant C\|f\|_{2} \text {. }
$$

Received by the editors August 12, 1980 and, in revised form, October 27, 1982. 1980 Mathematics Subject Classification. Primary 42A45, 47B38; Secondary 10G10.

Key words and phrases. Operator, weak restricted type $(2,2)$, commuting with translation, convolution operator, multiplier.

'This research was supported by National Science Foundation Grant MCS 76-81747 A01. 
Say that $T$ is of weak type $(2,2)$ and write $T \in w .(2,2)$ if $(1.4)$ holds for all $f$ in $L^{2}(G)$. Say that $T$ is of restricted weak type $(2,2)$ and write $T \in w . r .(2,2)$ if $(1.4)$ holds whenever $f$ is the characteristic function of a measurable set. From the definitions we see

$$
r .(2,2) \supset s .(2,2) \text { and w.r. }(2,2) \supset w .(2,2) \text {. }
$$

From Tchebyshev's inequality (1.3) and the definitions we also have immediately that $w .(2,2) \supset s .(2,2)$ and $w . r .(2,2) \supset r .(2,2)$.

If $G=\mathbf{R}$, call $B(x)=B_{\alpha, \beta, k}(x)$ a battlement if there are real numbers $\alpha<\beta$ and a positive integer $k$ such that $B(x)=1$ on the intervals

$$
\begin{aligned}
I_{j} & =\left(\alpha+\frac{2(j-1)}{2 k-1}(\beta-\alpha), \alpha+\frac{2 j-1}{2 k-1}(\beta-\alpha)\right) \\
& =\left(\alpha_{j}, \beta_{j}\right), \quad j=1,2, \ldots, k,
\end{aligned}
$$

and $B(x)=0$ otherwise (see Figure 1). If $G=\mathbf{T}$, add the conditions that $0 \leqslant \alpha$ and $\beta \leqslant 1$; while if $G=\mathbf{Z}$, add the condition that $x$ must be an integer in one of the $I_{j}$ for $B(x)$ to be 1 .

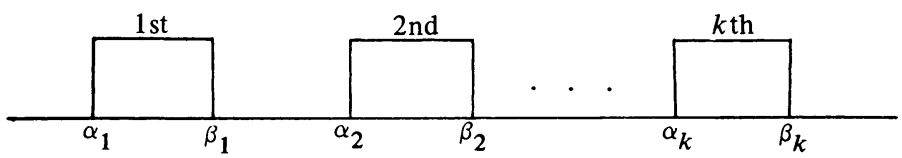

FIGURE 1

If (1.1) holds whenever $f$ is a battlement, say that $T$ is of very restricted type $(2,2)$ and write $T \in v . r .(2,2)$; if only (1.4) holds then say $T$ is of very weak restricted type $(2,2)$ and write $T \in v \cdot w \cdot r .(2,2)$. As above, we have the trivial inclusions

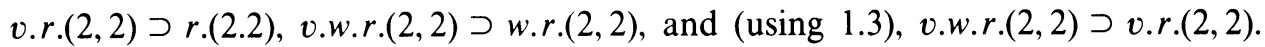
All the relations that have been mentioned are summarized in Figure 2 where $A$ $\rightarrow B$ means that whenever an operator is of type $A(2,2)$ then it is also of type $B(2,2)$.

Michael Cowling has shown that the reverse implication w.r. implies $s$. (see (2) of Figure 2) holds provided $T$ commutes with translation. His result holds whenever $G$ is an amenable group [2]. For the purposes of this paper, we note that $\mathbf{Z}, \mathbf{T}$ and $\mathbf{R}$ are all amenable. In a sense, this result says that the family of all characteristic functions of sets is a testing set-if an operator is well behaved on this family, then it is well behaved on all $L^{2}$ functions. Among candidates for testing sets "smaller is better". The inadequacy of the family of all characteristic functions of intervals as a testing set (see [1]; the referee observes that P. M. Knopf, Weak-type multipliers, Studia Math 67 (1980), 73-84 contains further examples) led to trying battlements as a testing set. The major results of this paper are that for $T$ commuting with translation, v.r. implies $s$. when $G=\mathbf{R}$ or $\mathbf{T}$ (Theorems 1-3), but that $v . r$. need not imply $s$. if $G=\mathbf{Z}$ (Theorem 6) (see (1) of Figure 2).

Cowling's w.r. implies $s$. theorem for $T$ (indeed for any compact abelian group) is part of the folklore but we include the proof to make the point that it is easy for such groups. The same theorem for $\mathbf{R}$ (Theorem 5) is more interesting, so we give a new proof of Cowling's theorem specialized to $\mathbf{R}$ along the lines of a great paper of Karel de Leeuw (see [4]) and a discussion of its relation to Cowling's proof. 


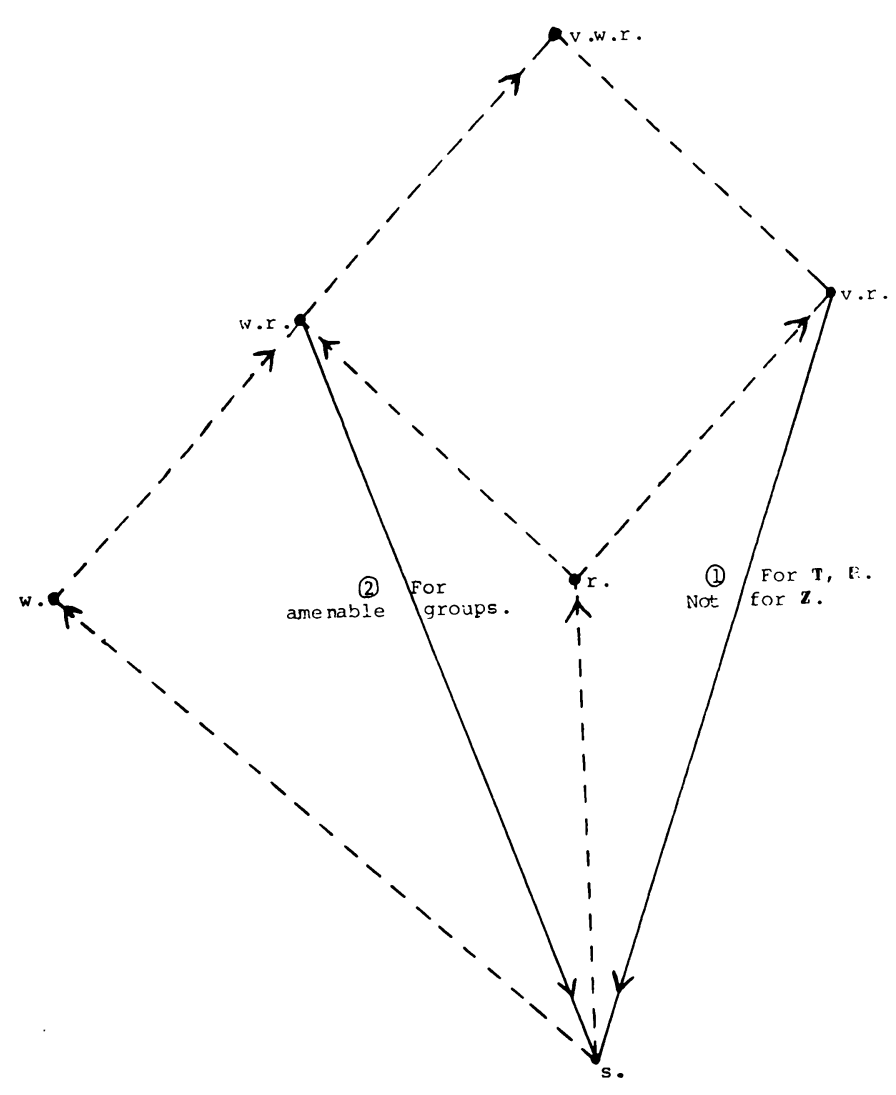

FIGURE 2

At the end of $\S 3$ we indicate why none of the 7 trivial implications of Figure 2 may be reversed if the hypothesis of commuting with translation is removed.

In $\$ 4$ we give an application of these results with an analytic number theoretic flavor (Theorem 7) and suggest some further research.

All of the positive results (Theorems 1-5) are peculiar to $p=2$. M. Zafran has shown that $w$. does not imply $s$. for $p<2$ (see [8]). By duality $r$. does not imply $s$. for $p>2$, so w.r. does not imply $s$. for any $p \neq 2$.

\section{Positive results.}

THEOREM 1. Let $T$ commute with translation. The following are equivalent if $G=\mathbf{R}$ or $\mathbf{T}$ :

(i) $T$ is of strong type $(2,2)$,

(ii) $T$ is of very restricted type $(2,2)$,

(iii) $T$ is of weak restricted type $(2,2)$.

Implications (i) $\Rightarrow$ (ii) and (i) $\Rightarrow$ (iii) are trivial as mentioned above. We break the proof of (ii) $\Rightarrow$ (i) into Theorems 2 and 3; and the proof of (iii) $\Rightarrow$ (i) into Theorems 4 and 5. 
THEOREM 2. Let the linear operator $S$ commute with translation, be defined on $S F(\mathbf{R})$ -the set of all finite linear combinations of characteristic functions of intervals - and satisfy

$$
\|S b\|_{L^{2}(\mathbf{R})} \leqslant C\|b\|_{L^{2}(\mathbf{R})}
$$

for every battlement $b$. Then $S$ extends uniquely to $s$. with $(2,2)$ operator norm $\leqslant \pi C / \sqrt{2}$.

Define a complex-valued function on $\mathbf{R}$ by

$$
\hat{R}(\xi)=\left(S \chi_{(0,1)}\right) \hat{(}(\xi) / \hat{\chi_{(0,1)}}(\xi)
$$

where we define the Fourier transform by

$$
\hat{f}(\xi)=\int_{-\infty}^{\infty} f(x) e^{-2 \pi i x \xi} d x
$$

and $\chi_{(0,1)}$ is the characteristic function of the unit interval. Note that $\hat{\chi}_{(0,1)}(\xi) \neq 0$ a.e.

Our method of proof will be to first show that the operator mapping $f$ to $(S f)$ agrees with the multiplier operator $m_{R}: f \rightarrow \hat{R} \cdot \hat{f}$ on $S F$ and to then show that the multiplier function $\hat{R}$ is a bounded function.

Lemma 1. For $f \in S F, \hat{R} \hat{f} \equiv(S f)$.

Proof. Linearity considerations show that it suffices to prove the lemma for $f=\chi_{(a, b)}$ - the characteristic function of an interval. We start with $a=0, b=1 / \mathrm{m}$, where $m>0$ is an integer. Use the facts that the Fourier transform converts a translation into multiplication by an exponential- $\left(f_{\tau} \hat{)}(\xi)=e^{-2 \pi i \tau \xi} \hat{f}(\xi)\right.$ where $f_{\tau}(x)=f(x-\tau)$ - to obtain

$$
\begin{aligned}
\hat{\chi}_{(0,1)}(\xi) & \equiv \sum_{k=0}^{m-1} \hat{\chi}_{(k / m,(k+1) / m)}(\xi)=\sum_{k=0}^{m-1}\left[\left(\chi_{(0,1 / m)}\right)_{k / m}\right]^{\wedge}(\xi) \\
& =\left(\sum_{k=0}^{m-1} e^{-2 \pi i k \xi / m}\right) \hat{\chi}_{(0,1 / m)}(\xi) .
\end{aligned}
$$

Since $S$ commutes with translation we have similarly that

$$
\left(S \chi_{(0,1)}\right)^{\hat{n}}(\xi)=\left(\sum_{k=0}^{m-1} e^{-2 \pi i k \xi / m}\right)\left(S \chi_{(0,1 / m)}\right)^{\hat{n}}(\xi),
$$

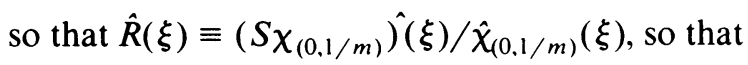

$$
\hat{R} \hat{\chi}_{(0,1 / m)} \equiv\left(S \chi_{(0,1 / m)}\right) \hat{.}
$$

Since $R$ is given as a multiplier it also commutes with translations, so from this identity follows

$$
\hat{R} \hat{\chi}_{(k / m,(k+1) / m)} \equiv\left(S \chi_{(k / m,(k+1) / m)}\right) \hat{.}
$$

From this, the identity

$$
\chi_{(k / m, l / m)} \equiv \sum_{j=k}^{l-1} \chi_{(j / m,(j+1) / m)} \quad \text { for } k<l
$$


and the linearity of both $S$ (assumed) and $m_{R}$ (see 2.2), it follows that

$$
\hat{R} \hat{\chi}_{(a, b)} \equiv\left(S \chi_{(a, b)}\right)^{\hat{n}} \quad \text { for } a, b \text { rational. }
$$

Passing to the general case, let $\chi$ be the characteristic function of an interval $(a, b)$ and let $\left\{\chi_{n}\right\}$ be a sequence of characteristic functions of intervals with rational endpoints tending to $a$ and $b$. Since $\left|\chi-\chi_{n}\right|$ is a sum of 2 characteristic functions of (short) intervals, by Plancherel's formula and (2.1) we have

$$
\left\|(S \chi)^{\hat{n}}-\left(S \chi_{n}\right) \hat{\|}_{2}=\right\| S \chi-S \chi_{n}\left\|_{2} \leqslant \sqrt{2} C\right\| \chi_{n}-\chi \|_{2} \rightarrow 0 .
$$

From this, (2.2) and (2.4), $\hat{R} \hat{\chi}_{n} \rightarrow(S \chi) \hat{)}$ in $L^{2}$, so for some subsequence $\left\{n_{k}\right\}$,

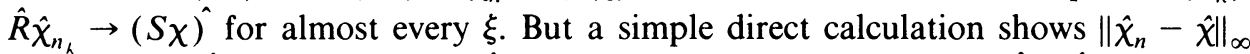
$\rightarrow 0$ so that $\hat{R} \hat{\chi}_{n_{k}}$ also tends to $\hat{R} \hat{\chi}$ almost everywhere, whence $(S \chi) \hat{X} \hat{R} \hat{\chi}$.

LEMMA 2. If $S$ is defined as a multiplier from $S F$ to $L^{2}(\mathbf{R})$ and satisfies

$$
\|S(b)\|_{2}=\|\hat{S} \cdot \hat{b}\|_{2} \leqslant C\|b\|_{2}
$$

for every battlement $b$, then $\|\hat{S}\|_{\infty} \leqslant \pi C / 2$.

REMARK. The idea of the proof is to find a "frequency" $\alpha$ at which $\hat{S}$ "reaches" its maximum $M$ and then to let the battlement $b$ "be" the pure exponential $e^{i \alpha x}$ so that $\hat{S}(\xi) \hat{b}(\xi)$ " $=" M \cdot \delta(\xi-\alpha)$, where $\delta$ is the Dirac delta function. Taking the inverse Fourier transform shows that the operator $S$ has stretched the norm of $b$ by $M$. The proof which follows was sharpened and shortened by an expert who read a preprint.

Proof. A simple computation shows

$$
\left\|\chi_{(0,1 / 2 \alpha)}\right\|_{2}=\sqrt{\frac{1}{2 \alpha}}=\left\|\hat{\chi}_{(0,1 / 2 \alpha)}\right\|_{2}
$$

and

$$
\left|\hat{\chi}_{(0,1 / 2 \alpha)}(\xi)\right|=\left|\frac{\sin (\pi \xi / 2 \alpha)}{\pi \xi}\right| .
$$

Let $b=\chi_{(0,1 / 2 R)}$, and substitute (2.7) and (2.8) into (2.6) to get

$$
\begin{aligned}
\left(\frac{1}{\pi R}\right)^{2} \int_{-R}^{R}|\hat{S}(\xi)|^{2} d \xi & \leqslant \int_{-R}^{R}|\hat{S}(\xi)|^{2}\left(\frac{\sin (\pi \xi / 2 R)}{\pi \xi}\right)^{2} d \xi \\
& \leqslant \int_{-\infty}^{\infty} \cdots d \xi \leqslant C \cdot \frac{1}{2 R}
\end{aligned}
$$

whence $|\hat{S}|^{2}$ is a locally integrable function. Let $\alpha$ be any Lebesgue point of $|\hat{S}|^{2}$ so that as $t \rightarrow 0$,

$$
\left.\frac{1}{2 t} \int_{-t}^{t}|| \hat{S}(\alpha+s)\right|^{2}-|\hat{S}(\alpha)|^{2} \mid d s=\varepsilon(t) \rightarrow 0
$$

Let $b=\sum_{k=0}^{N-1} \chi_{(k / \alpha, k / \alpha+1 / 2 \alpha)}$. By direct calculation

$$
\begin{gathered}
\|b\|_{2}^{2}=\frac{N}{2 \alpha,} \\
\hat{b}(\xi)=\hat{\chi}_{(0,1 / 2 \alpha)}(\xi) \sum_{k=0}^{N-1} e^{-2 \pi i k \xi / \alpha}
\end{gathered}
$$


and

$$
\left|\sum_{k=0}^{N-1} e^{-2 \pi i k \xi / \alpha}\right|=\left|\frac{\sin (\pi N \xi / \alpha)}{\sin (\pi \xi / \alpha)}\right| .
$$

Putting (2.8), (2.11), (2.12) and (2.13) into (2.6) gives

$$
\int_{-\infty}^{\infty}|\hat{S}(\xi)|^{2}\left(\frac{\sin (\pi \xi / 2 \alpha)}{\pi \xi}\right)^{2}\left(\frac{\sin (\pi N \xi / \alpha)}{\sin (\pi \xi / \alpha)}\right)^{2} d \xi \leqslant C^{2} \cdot\left(\frac{N}{2 \alpha}\right)
$$

Making a change of variable $\xi=\alpha+\alpha t / \pi N, d \xi=\alpha d t / \pi N$ and noting $|\sin \theta|=$ $|\sin (N \pi+\theta)|$ yields

$$
\int_{-\infty}^{\infty}\left|\hat{S}\left(\alpha+\frac{\alpha t}{\pi N}\right)\right|^{2}\left\{\frac{1}{\pi}\left(\frac{\sin \pi(1+t / \pi N) / 2}{1+t / \pi N}\right)^{2}\left(\frac{\sin t}{N \sin (t / N)}\right)^{2}\right\} d t \leqslant \frac{\pi^{2}}{2} C^{2} .
$$

The proof is now formally completed by letting $N \rightarrow \infty$ inside the integral getting

$$
|\hat{S}(\alpha)|^{2}=|\hat{S}(\alpha)|^{2} \int_{-\infty}^{\infty} \frac{1}{\pi}\left(\frac{\sin t}{t}\right)^{2} d t \leqslant\left(\frac{\pi}{\sqrt{2}}\right)^{2} C^{2} .
$$

To complete the proof we must justify the passage from (2.15) to (2.16) at almost every $\alpha$. The set of Lebesgue points has full measure so we fix one, say $\alpha$ again, and show that (2.16) holds at $\alpha$.

Since $|\sin N \theta| \leqslant N|\sin \theta|(\operatorname{see}[9$, p. 71]) and $|\sin \theta / \theta| \leqslant 1$, we have

$$
k_{N}(t) \leqslant \pi / 4 \text {, }
$$

where $k_{N}(t)$ denotes the expression in curly brackets in inequality (2.15).

Fix $R$ very large. Then from (2.10), (2.17) and (2.15) we get

$$
\begin{aligned}
|\hat{S}(\alpha)|^{2} \int_{-R}^{R} k_{N}(t) d t \leqslant & \left.\int_{-R}^{R}|| \hat{S}\left(\alpha+\frac{\alpha t}{\pi N}\right)\right|^{2}-|\hat{S}(\alpha)|^{2} \mid k_{N}(t) d t \\
& +\int_{-\infty}^{\infty}\left|\hat{S}\left(\alpha+\frac{\alpha t}{\pi N}\right)\right|^{2} k_{N}(t) d t \\
& \leqslant 2 R \varepsilon\left(\frac{\alpha R}{\pi N}\right) \cdot \frac{\pi}{4}+\frac{\pi^{2}}{2} C .
\end{aligned}
$$

Letting $N \rightarrow \infty$ gives

$$
|\hat{S}(\alpha)|^{2} \frac{1}{\pi} \int_{-R}^{R}\left(\frac{\sin t}{t}\right)^{2} d t \leqslant \frac{\pi^{2}}{2} C .
$$

Letting $R \rightarrow \infty$ gives (2.16) and completes the proof.

Continuing the proof of Theorem 1 we now pass to the case $G=\mathbf{T}$.

THEOREM 3. Let the linear operator T commute with translation, be defined on $S F(\mathbf{T})$ and be of very restricted type $(2,2)$ with operator norm $C$; so that

$$
\|T b\|_{2} \leqslant C\|b\|_{2}
$$

for every battlement $b$. Then $T$ extends uniquely to $s$. with $(2,2)$ operator norm $\leqslant \pi C / \sqrt{2}$. 
Proof. As in the proof of Lemma 1 we first define a multiplier $S$ which agrees with $T$ on the function $\chi_{0}=\chi_{[0,1 / 2 \pi]}$. Explicitly, $\chi_{0}(x)=\sum c_{n} e^{2 \pi i n x}$ where

$$
c_{n}=\frac{\sin (n / 2)}{\pi n} e^{-i n / 2} \neq 0 \text { for all } n\left(c_{0}=(2 \pi)^{-1}\right),
$$

so if $T \chi_{0}(x)=\sum\left(T \chi_{0}\right) \hat{(n)} e^{-2 \pi i n x}$, define $S$ by $S\left(\sum a_{n} e^{2 \pi i n x}\right)=\sum t_{n} a_{n} e^{2 \pi i n x}$ where $t_{n}=\left(T \chi_{0}\right) \hat{(n) / c_{n}}$. Computations like those in the proof of Lemma 1 show $S$ and $T$ to agree on characteristic functions of intervals whose endpoints are rational multiples of $1 / 2 \pi$.

An approximation argument similar to the one given in Lemma 1 and using (2.18) shows $T$ to agree with $S$ on the characteristic function of any interval. Next we note that $S$ and $T$ agree on all battlements since both are linear.

To complete the proof we need to show that $\left|t_{n}\right| \leqslant \pi C / \sqrt{2}$ for every integer $n$. Fix any integer $n \neq 0$. The battlement

$$
b=\sum_{\nu=1}^{|n|} \chi_{(\nu /|n|-1 / 4|n|, \nu /|n|+1 / 4|n|)}
$$

almost "is" the character $e^{2 \pi i n x}$. More precisely, $\left\|e^{2 \pi i n x}\right\|_{2}=\mid\left(e^{2 \pi i n x} \hat{)}(n) \mid=1\right.$, while

$$
\|b\|_{2}=\frac{\pi}{\sqrt{2}}|\hat{b}(n)|
$$

Equality (2.20) is an elementary computation. Finally, combine (2.18) and (2.20) to get

$$
\left|t_{n}\right||\hat{b}(n)|=|(T b) \hat{(}(n)| \leqslant\|T b\|_{2} \leqslant C\|b\|_{2}=\frac{\pi}{\sqrt{2}} C|\hat{b}(n)|
$$

where the first inequality is the estimate of a Fourier coefficient by the $L^{2}$ norm of the function. Dividing (2.21) by $|\hat{b}(n)|$ completes Theorem 3. (Note that if $n=0$, the same argument with $b(x)=1$ yields $\left|t_{0}\right| \leqslant C \leqslant \pi C / \sqrt{2}$.)

THEOREM 4. Let $G=\mathbf{T}$. Let the linear operator $T$ commute with translation, and be weak restricted $(2,2)$ with

$$
\|T \chi\|_{2, \infty}^{*} \leqslant C\|\chi\|_{2}
$$

whenever $\chi$ is a characteristic function. Then $T$ extends uniquely to $s$. with $(2,2)$ operator norm bounded by $8 C$.

The proofs of Theorems 4 and 5 will use much of the theory of $L_{p q}$ spaces. See either Hunt's long and clear paper [3] or Chapter 5, $\$ 3$ of [6] for the basic definitions and facts concerning these spaces.

The proof of Theorem 4 pivots around the linearity of $\|\cdot\|_{2,1}^{*}$. It belongs to the folklore, but is included here to show why the constant expands to $8 \mathrm{C}$. 
Proof of Theorem 4. If $\chi$ is the characteristic function of a set, then $\|T \chi\|_{2, \infty}^{*} \leqslant$ $C\|\chi\|_{2}=C\|\chi\|_{2,1}^{*}$. If $f$ is a positive simple function, write $f=\sum f_{i}$ where the $f_{i}$ are positive multiples of characteristic functions of sets chosen so that $f^{*}=\sum f_{i}^{*}[6, \mathrm{p}$. 194]. Then

$$
\begin{aligned}
\|T f\|_{2, \infty}^{*} & \leqslant\left\|\sum\left(T f_{i}\right)\right\|_{2, \infty} \leqslant \sum\left\|T f_{i}\right\|_{2, \infty} \leqslant 2 \sum\left\|T f_{i}\right\|_{2, \infty}^{*} \leqslant 2 C \sum\left\|f_{i}\right\|_{2} \\
& =2 C \sum\left\|f_{i}\right\|_{2,1}^{*}=2 C\|f\|_{2,1}^{*} .
\end{aligned}
$$

A general simple function $f$ may be decomposed into $f_{1}-f_{2}+i\left(f_{3}-f_{4}\right)$ where each $f_{i}$ is positive and $\operatorname{supp} f_{1} \cap \operatorname{supp}\left(f_{2}\right)=\operatorname{supp}\left(f_{3}\right) \cap \operatorname{supp}\left(f_{4}\right)=\varnothing$. Apply (2.22) to each $f_{i}$ and observe that $f^{*}(t)$ dominates each $f_{i}^{*}(t)$ to get

$$
\|T f\|_{2, \infty}^{*} \leqslant 2 C \sum_{i=1}^{4}\left\|f_{i}\right\|_{2,1}^{*} \leqslant 8 C\|f\|_{2,1}^{*}
$$

for all simple functions and, extending $T$ by continuity, for all functions in $L(2,1)$. Since $T$ is linear, bounded, and commutes with translation, it is easy to see that $T$ acts as a multiplier, i.e., there is a sequence of numbers $\{\hat{T}(n)\}$ such that if $f \sim \sum c_{n} e^{2 \pi i n x} \in L(2,1)$, then $T f \sim \sum \hat{T}(n) c_{n} e^{2 \pi i n x}$. (To see this, apply $T$ successively to individual characters, finite linear combinations of characters, and limits of these.) Finally, $T$ does take $L_{2}$ boundedly into $L_{2}$ since for every $n$,

$$
|\hat{T}(n)|=\left\|\hat{T}(n) e^{2 \pi i n x}\right\|_{2, \infty}^{*}=\left\|T\left(e^{2 \pi i n x}\right)\right\|_{2, \infty}^{*} \leqslant 8 C\left\|e^{2 \pi i n x}\right\|_{2,1}^{*}=8 C
$$

where the inequality follows from (2.23).

The proof of Theorem 1 now requires only the implication (iii) $\Rightarrow$ (i) in the case of $G=\mathbf{R}$. This was proved by Michael Cowling in [2]. His proof was given for $G$ any amenable group, and hence holds for a vastly more general situation than we are treating. We will present here a new proof. We will also comment on the relation between this proof and Cowling's proof specialized (by Cowling in a private communication to the author) to $\mathbf{R}$.

THEOREM 5. If $T$ commutes with translation and is of weak restricted type $(2,2)$ over $\mathbf{R}$, then $T$ is of strong type $(2,2)$.

Proof. As in Theorem 4, we may assume that $T$ maps $L(2,1)(\mathbf{R})$ boundedly into $L(2, \infty)(\mathbf{R})$ :

$$
\|T f\|_{2, \infty}^{*} \leqslant A\|f\|_{2,1}^{*} .
$$

Since $T$ is determined by its restriction to $C_{c}^{\infty}$ (infinitely differentiable compactly supported functions) which is mapped into $L(2, \infty)$ - a space of distributions; by the Schwartz Kernel theorem, $T$ is given by convolution with a distribution: $T f=\phi * f$, say. By regularizing, we may assume that $\phi$ is a $C^{\infty}$ function. (Explicitly, let $\delta \geqslant 0$ be $C_{c}^{\infty}, \int \delta=1$, so that $\left\{\delta_{n}\right\}=\{n \delta(n x)\}_{n=1,2, \ldots}$ is an approximate identity 
and let $\phi_{n}=\phi * \delta_{n}$. Then $\phi_{n}$ is a $C^{\infty}$ function. Since

$$
\|f * g\|_{2,1}^{*} \leqslant\|f\|_{1}\|g\|_{2,1}^{*}
$$

which is easy to see from writing $\|f * g\|_{2,1}^{*}=\sup _{\|h\|_{2, \infty}} \leqslant 1\left|\int(f * g) h\right|$, from (2.24) we have

$$
\left\|\phi_{n} * f\right\|_{2, \infty}^{*}=\left\|\phi *\left(\delta_{n} * f\right)\right\|_{2, \infty}^{*} \leqslant A\left\|\delta_{n} * f\right\|_{2,1}^{*} \leqslant A\|f\|_{2,1}^{*}
$$

which is (2.23) for $\phi_{n}$. If from 2.26 we can prove that $\phi_{n} *$ satisfies $\left\|\phi_{n} * f\right\|_{2} \leqslant A\|f\|_{2}$, it will follow that $\hat{\phi}_{n}$-the Fourier transform of $\phi_{n}$ in the sense of distributions is actually a function essentially bounded by $A$. Then for any $g \in L^{1}|(\hat{\phi}, g)|=$ $\lim _{n \rightarrow \infty}\left|\left(\hat{\phi}_{n}, g\right)\right| \leqslant A\|g\|_{1}$ by Lebesgue's dominated convergence theorem so that $\hat{\phi}$ is also a function essentially bounded by $A$, whence $T$ is of strong type $(2,2)$.)

In a beautiful paper [4] Karel de Leeuw characterized multipliers on $L^{p}$ and then used this characterization to transfer an operator bounded on $L^{p}(\mathbf{R})$ to one bounded on $L^{p}(\hat{\mathbf{D}})$ where $\hat{\mathbf{D}}$, the Bohr group of $\mathbf{R}$, is compact. Our proof of Theorem 5 is almost immediate from the following slight generalization of de Leeuw's characterization.

LEMMA 3. Let $m$ be a tempered distribution and $M$ be the operator given by convolution with the inverse Fourier transform of $m$. Then the following are equivalent:

1. $M$ extends to a bounded operator from $L_{2,1}(\mathbf{R})$ to $L_{2, \infty}(\mathbf{R})$ with finite operator norm

$$
\|M\|=\sup _{f \in L_{2,1}} \frac{\|M(f)\|_{2, \infty}}{\|f\|_{2,1}^{*}} ;
$$

2. there is a constant $K$ so that

$$
|m(\phi \cdot \psi)| \leqslant K\|\hat{\phi}\|_{2,1}^{*}\|\hat{\psi}\|_{2,1}^{*}
$$

for all $\phi$ and $\psi$ in $\delta$ - the Schwartz space of infinitely differentiable rapidly decreasing functions.

If 1 and 2 hold, then $\|M\|$ is the smallest constant $K$ for which the inequality (2.27) is valid for all $\phi$ and $\psi$ in $\delta$.

ObServations. By $\|f\|_{2, \infty}$, we mean $\sup _{t>0} t^{-1 / 2} \int_{0}^{t} f^{*}(s) d s$. Unlike the norm $\|\cdot\|_{2, \infty}^{*}$, the norm $\|\cdot\|_{2, \infty}$ satisfies the triangle inequality. Furthermore, we have $\|\psi\|_{2, \infty}^{*} \leqslant\|\psi\|_{2, \infty} \leqslant 2\|\psi\|_{2, \infty}^{*}[6$, p. 204]. We use the unstarred norm here because of its compatibility with the $L_{p, q}$ versions of Hölder's inequality. Explicitly, G. G. Lorentz has shown, for $f \in L_{2,1}$ and $g \in L_{2, \infty}$,

$$
\left|\int_{-\infty}^{\infty} f g\right| \leqslant\|f\|_{2,1}^{*}\|g\|_{2, \infty} \quad(\text { see }[5]) .
$$

It is not difficult to prove the following converse:

$$
\|\psi\|_{2, \infty}=\sup _{\phi \in \delta} \frac{\left|\int_{-\infty}^{\infty} \phi \psi\right|}{\|\phi\|_{2,1}^{*}} .
$$


Proof of Lemma 3. We have

$$
\begin{aligned}
& \|M\|=\sup _{f \in L_{2,1}} \frac{\|M f\|_{2, \infty}}{\|f\|_{2,1}^{*}} \quad \begin{array}{l}
\text { (the observations justify using }\|\cdot\|_{2,1}^{*} \text { and } \\
\|\cdot\|_{2, \infty} \text { in this definition), }
\end{array} \\
& =\sup _{\phi \in \mathcal{S}} \frac{\|M(\check{\phi})\|_{2, \infty}}{\|\check{\phi}\|_{2,1}^{*}} \quad \begin{array}{l}
\left(\text { since } \mathcal{S} \text { is dense in } L_{2,1}\right. \text { and the inverse } \\
\text { Fourier transform is an isometry on } \mathcal{S}),
\end{array} \\
& =\sup _{\phi, \psi \in \mathcal{S}} \frac{\left|\int M(\check{\phi}) \hat{\psi}\right|}{\|\hat{\phi}\|_{2,1}^{*}\|\hat{\psi}\|_{2,1}^{*}} \quad \begin{array}{l}
\text { (by }(2.29), \check{\phi}(x)=\hat{\phi}(-x) \text { and }\|\hat{\phi}(-x)\|_{2,1}^{*} \\
\left.=\|\hat{\phi}\|_{2,1}^{*}\right),
\end{array} \\
& \text { (by definition } M(\check{\phi})=\check{m} * \check{\phi} \text { so } \int(M(\check{\phi})) \hat{\psi} \\
& =(\check{m} * \check{\phi})(\hat{\psi})=\check{m}(\tilde{\dot{\phi}} * \hat{\psi})
\end{aligned}
$$

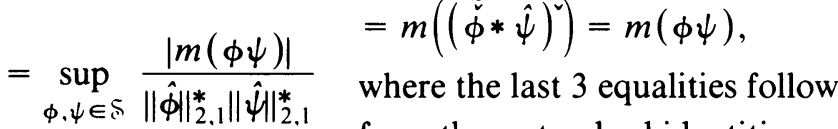

$$
\begin{aligned}
& \text { from these standard identities and } \\
& \text { definitions: } m(\check{f})=\check{m}(f), \tilde{\phi}(x)=\phi(-x), \hat{\tilde{\phi}}=\tilde{\hat{\phi}} \\
& \left.(m * n)(f)=m(\tilde{n} * f) \text {, and }(\phi * \psi)^{\nu}=\tilde{\hat{\phi}} \cdot \tilde{\psi}\right) \text {. }
\end{aligned}
$$

The first supremum in this string of equalities is finite if and only if the last one is, which proves Lemma 3.

Proof of Theorem 5. We may assume that the operator $T$ is given by convolution with a distribution $\check{m}$ and acts boundedly from $L_{2,1}$ to $L_{2, \infty}$ :

$$
\|T f\|_{2, \infty}=\|\check{m} * f\|_{2, \infty} \leqslant K\|f\|_{2,1}^{*} .
$$

Let $\phi^{\varepsilon}(x)=(\sqrt{2 \pi \varepsilon})^{-1} e^{-x^{2} / 2 \varepsilon}$ be the approximate identity arising from the normal curve. Some easily computed properties of $\phi^{\varepsilon}$ are

$$
\begin{gathered}
\left(\sqrt{\phi^{\varepsilon}}\right)^{\wedge}(x)=\sqrt[4]{8 \pi \varepsilon} e^{-4 \varepsilon \pi^{2} x^{2}}, \\
{\left[\left(\sqrt{\phi^{\varepsilon}}\right)^{\wedge}\right]^{*}(t)= \begin{cases}\left(\sqrt{\phi^{\varepsilon}}\right)^{-}\left(\frac{t}{2}\right), & t \geqslant 0, \\
0, & t<0,\end{cases} }
\end{gathered}
$$

and

$$
\left\|\left(\sqrt{\phi^{\varepsilon}}\right)^{\hat{n}}\right\|_{2,1}^{*}=\left(\frac{1}{2 \pi}\right)^{1 / 4} \int_{0}^{\infty} u^{-1 / 2} e^{-u^{2}} d u=\left(\frac{1}{32 \pi}\right)^{1 / 4} \Gamma\left(\frac{1}{4}\right) .
$$

From (2.30) and the characterizing Lemma 3, we have for any $\phi$ and $\psi \in \mathcal{S}$,

$$
|m(\phi \psi)| \leqslant K\|\hat{\phi}\|_{2,1}^{*}\|\hat{\psi}\|_{2,1}^{*} .
$$

Substitute $\phi(x)=\psi(x)=\sqrt{\phi^{\varepsilon}(x-\tau)}=\left(\sqrt{\phi^{\varepsilon}(x)}\right)_{\tau}$ and use

$$
\left|\left(f_{\tau}(x)\right) \hat{\mid}=\right| \hat{f}(x) e^{-2 \pi i x \tau}|=| \hat{f}(x) \mid
$$

and (2.31) to get

$$
\left|\left(m * \phi^{\varepsilon}\right)(\tau)\right|=\left|m\left(\left(\phi^{\varepsilon}\right)_{\tau}\right)\right| \leqslant K\left[\left(\frac{1}{32 \pi}\right)^{1 / 4} \Gamma\left(\frac{1}{4}\right)\right]^{2}=\frac{1}{\sqrt{32 \pi}} \Gamma\left(\frac{1}{4}\right)^{2} \cdot K .
$$


Letting $\varepsilon \rightarrow 0$ as in the first proof shows $m$ to be a function with $L^{\infty}$ norm no more than $c .=(1 / \sqrt{32 \pi}) \Gamma(1 / 4)^{2} \doteq 1.3110287$ times the original operator norm. Thus the original mapping $T f=(m \cdot \hat{f})$ is given as multiplication by the bounded function $m$ and hence extends to a bounded operator from $L^{2}$ to $L^{2}$.

Michael Cowling's proof involves defining $U_{n}$ to be an even $C^{\infty}$ function supported in $\left[-\left(n^{2}+n+1\right), n^{2}+n+1\right]$ rising from 0 to $a=1 /\left(2 n^{2}+1\right)^{1 / 2}$ on $\left(-n^{2}-n-1,-n^{2}-n\right)$ and constantly equal to $a$ on $\left[-n^{2}-n, n^{2}+n\right]$; and $V_{n}$ to be an even $C^{\infty}$ function supported in $\left[-n^{2}-1, n^{2}+1\right]$ equal to $a$ on $\left[-n^{2}, n^{2}\right]$ and rising from 0 to $a$ on $\left(-n^{2}-1,-n^{2}\right)$ in such a way that $\int V_{n}=\left(2 n^{2}+1\right)^{1 / 2}$. Then $\left\{\hat{U}_{n} \cdot \hat{V}_{n}\right\}_{n=1,2, \ldots}$ are an approximate identity and play the same sort of role in Cowling's proof that $\left\{\sqrt{\phi^{\varepsilon}} \cdot \sqrt{\phi^{\varepsilon}}\right\}_{\varepsilon}>0$ plays in ours.

3. Negative results. On the integers $\mathbf{Z}$ a battlement $b$ is the characteristic function of a set of the form $\{m, m+1, \ldots, m+k-1 ; m+2 k, m+2 k+1, \ldots, m+2 k$ $+k-1 ; m+4 k, \ldots ; \ldots ; m+2(l-1) k, m+2(l-1) k+1, \ldots, m+2(l-1) k$ $+k-1\}$, so that

$$
\hat{b}(x)=\sum_{j=0}^{l-1} \sum_{n=0}^{k-1} e^{(m+2 j k+n) 2 \pi i x}, \quad 0 \leqslant x \leqslant 1 .
$$

Although mapping battlements on $G$ boundedly from $L^{2}(G)$ to $L^{2}(G)$ forces a linear operator commuting with translation to be bounded on all of $L^{2}(G)$ when $G$ is $\mathbf{R}$ or $\mathbf{T}$ (see §2, Theorem 2), this is not the case when $G=\mathbf{Z}$.

THEOREM 6. There is a convolution operator $K$ defined from $S F(Z)=($ Trigonometric polynomials) to $l^{2}=L^{2}(\mathbf{Z})=L^{2}(\mathbf{T})$ which satisfies $\|K * b\|_{2} \leqslant\|b\|_{2}$ for every battlement $b$, but which does not extend continuously to a bounded operator on $l^{2}$.

LEMMA 4. Given $\varepsilon>0$, there is an interval $I,|I| \leqslant \varepsilon$, such that for every battlement

$$
\hat{b}(t)=\sum_{j=0}^{l-1} \sum_{n=0}^{k-1} e^{(n+l j k+c) 2 \pi i t}, \quad\|\hat{b}\|_{2, I}=\left(\int_{I}|\hat{b}|^{2}\right)^{1 / 2} \leqslant \varepsilon\|b\|_{2} .
$$

Proof of Theorem. Let $\varepsilon_{i}=2^{-i-1}, i=1,2, \ldots$, and apply Lemma 4 , thereby producing $I_{i}$ such that $\left|I_{i}\right| \leqslant 2^{-i-1}$ and $\|b\|_{2, I} \leqslant 2^{-i-1}\|b\|_{2}$. Define $\hat{K}_{i}(t)=i, t \in I_{i}$, $\hat{K}_{i}=0$ otherwise and let $K=\Sigma\left(\hat{K}_{i}\right)$. Then

$$
\begin{aligned}
\|K * b\|_{2} & =\|\hat{K} \cdot \hat{b}\|_{2} \leqslant \sum\left\|\hat{K}_{i} \hat{b}\right\|_{2}=\sum i\|\hat{b}\|_{2, I_{i}} \\
& \leqslant\left(\sum i 2^{-i-1}\right)\|b\|_{2}=\|b\|_{2}
\end{aligned}
$$

but since $\hat{K}$ is unbounded, $K$ cannot be continuously extended to a bounded operator on all of $L^{2}(\mathbf{Z})$.

REMARK. $K \in L^{2}$ since $\|\hat{K}\|_{2}^{2} \leqslant \sum \int_{I_{i}} K_{i}^{2} \leqslant \sum i^{2} 2^{-i-1}<\infty$.

Proof Of Lemma 4. Let $\varepsilon>0$ be given. The required interval will be $I=[\alpha, \alpha+\beta]$ where $\alpha$ may be any irrational number in $\left(0, \frac{1}{4}\right)$ and the choice of $\beta$ will depend on $\varepsilon$. Fix such an $\alpha$. 
Since $\hat{b}$ is a sum of $k l$ exponentials (see 3.1) we have $\|\hat{b}\|_{2}^{2}=\int_{0}^{1}|\hat{b}|^{2}=k l$. An elementary computation shows

$$
|\hat{b}|=\left|\frac{\sin \pi k t}{\sin \pi t}\right| \cdot\left|\frac{\sin 2 \pi k l t}{\sin 2 \pi k t}\right|=\left|\frac{1}{\sin \pi t}\right|\left|\frac{\sin 2 \pi k l t}{2 \cos \pi k t}\right| .
$$

We need to show that

$$
\int_{\alpha}^{\alpha+\beta} \mid \hat{b}^{2} \leqslant \varepsilon \cdot\|\hat{b}\|_{2}^{2}=\varepsilon k l .
$$

If $\beta$ is so small that $\alpha+\beta<\frac{1}{4}$, then the integrand is increased by increasing the factor $|\sin \pi t|^{-2}$ to $\left|\frac{2}{\pi}(\pi t)\right|^{-2}$ so that (3.2) will follow if

$$
A=\frac{1}{16} \int_{\alpha}^{\alpha+\beta}\left|\frac{\sin 2 \pi k l t}{\cos \pi k t}\right|^{2} \frac{d t}{t^{2}}=\frac{k}{16} \int_{k \alpha}^{k \alpha+k \beta}\left|\frac{\sin 2 \pi l x}{\cos \pi x}\right|^{2} \frac{d x}{x^{2}} \leqslant \varepsilon k l .
$$

The estimation of $A$ depends on the size of $k$. If $k$ is large, say $k \geqslant k_{0}$ where $\pi^{2} / 32\left(\left[k_{0} \alpha\right]-1\right)<\varepsilon$, then we may be quite rough. In fact,

$$
A \leqslant \sum_{j=\left[k_{0} \alpha\right]}^{\infty} \frac{k}{16 j^{2}} \int_{j}^{j+1}\left|\frac{\sin 2 \pi l x}{\cos \pi x}\right|^{2} d x=\sum A_{j} .
$$

To estimate $A_{j}$, let $u=x-\left(j+\frac{1}{2}\right)$ and use the sine and cosine addition formulas to get

$$
A_{j}=\frac{k}{16 j^{2}} \int_{-1 / 2}^{1 / 2}\left(\frac{\sin 2 \pi l u}{\sin \pi u}\right)^{2} d u .
$$

Replace $\sin ^{2} \pi u$ by the smaller quantity $(2 u)^{2}$ and let $t=2 \pi l u$. We have

$$
A_{j} \leqslant \frac{2 \pi k l}{16 j^{2}} \int_{-\pi l}^{\pi l}\left(\frac{\sin t}{2 t}\right)^{2} d t<\frac{\pi k l}{32 j^{2}} \int_{-\infty}^{\infty}\left(\frac{\sin t}{t}\right)^{2} d t=\frac{\pi^{2} k l}{32 j^{2}},
$$

so that

$$
\begin{aligned}
A & \leqslant \sum A_{j} \leqslant \frac{\pi^{2}}{32} k l \sum_{j=[k \alpha]}^{\infty} \frac{1}{j^{2}}<\frac{\pi^{2}}{32} k l \int_{[k \alpha]-1}^{\infty} \frac{d x}{x^{2}} \\
& =\frac{\pi^{2}}{32} \frac{1}{[k \alpha]-1} k l<\varepsilon k l .
\end{aligned}
$$

We must still show that if $k \leqslant k_{0}$, a sufficiently small choice of $\beta$ will yield (3.3). The estimates $x^{-2} \leqslant(k \alpha)^{-2}$ and $\sin ^{2} 2 \pi l u \leqslant 1$ show that (3.3) will be established if we can get

$$
\frac{1}{16 k \alpha^{2}} \int_{k \alpha}^{k \alpha+k \beta} \frac{d x}{\cos ^{2} \pi x}<\varepsilon k l
$$

for all $k \leqslant k_{0}$ and all $l$. The set $T=\left\{k \alpha \mid 1 \leqslant k \leqslant k_{0}\right\}$ is a finite set of irrationals. Thus $\operatorname{dist}\left(T, \frac{1}{2} \mathbf{Z}\right)>0$. Hence if $\beta$ is sufficiently small, $\operatorname{dist}\left([k \alpha, k \alpha+k \beta], \frac{1}{2} \mathbf{Z}\right)$ is bounded away from 0 so that the integrands in (3.4) are uniformly bounded-say $1 /\left(\cos ^{2} \pi x\right)<M, k=1, \ldots, k_{0}$. Then choosing $\beta<16 \alpha^{2} \varepsilon / M$ proves (3.4). Lemma 4 , and hence Theorem 6 , is established. 
REMARKS. In Figure 2 there are seven trivial implications. Denoting operators of very weak restricted type $(2,2)$ by $v \cdot w . r$. etcetera, these implications are

$$
\left\{\begin{array}{l}
s . \text { implies } w ., \\
s . \text { implies } r ., \\
r . \text { implies } w . r ., \\
w . \text { implies } w . r ., \\
v . r . \text { implies } v \cdot w . r ., \\
r . \text { implies } v . r ., \text { and } \\
w . r . \text { implies } v . w . r .
\end{array}\right.
$$

If we drop the hypothesis of commuting with translation and consider linear operators in general, we find that none of these implications are reversible.

Let $\mathbf{T}=[0,1]$ and consider three operators

$$
\left(T_{1} f\right)(x)=\left(\int_{0}^{1} f(y) d y\right) x^{-1 / 2}, \quad\left(T_{2} f\right)(x)=\int_{0}^{1} y^{-1 / 2} f(y) d y
$$

for each $x$, and

$$
\left(T_{3} f\right)(x)=\left(\int_{0}^{1} y^{-1 / 2} f(y) d y\right) x^{-1 / 2}
$$

Assume that $T_{1}$ is defined on $L^{2}(\mathbf{T})$ and $T_{2}$ and $T_{3}$ are defined for step functions. Easy arguments show that $T_{1}$ is in $w$., but not $s$.; $T_{2}$ is in $r$., but cannot be extended to be in s.; and $T_{3}$ is in w.r., hence v.w.r., but cannot be extended to $w$. (see [7, pp. 282-284]). Letting $f$ be the battlement identically equal to 1 shows that $T_{3}$ is not in $v . r$., hence not in $r$. These examples, first given by Stein and Weiss [7, pp. 282-284], show the first 5 implications of (3.5) to be irreversible. The operator of Theorem 6 is in v.r., hence v.w.r. but neither in $r$. nor w.r. (for then by Theorem 1 it would extend to $s$.). Thus the last 2 implications of (3.5) are also irreversible.

\section{An application and questions.}

THEOREM 7. For each interval $I \subset[0,1]$, there is a sum of exponentials whose $L^{2}$ norm is concentrated on I. More precisely, there is an absolute constant $a_{0}>0$ such that for each interval $I \subset[0,1]$ there is a finite set $S=S(I) \subset \mathbf{Z}$ such that

$$
\int_{I}\left|\sum_{n \in S} e^{2 \pi i n x}\right|^{2} d x>a_{0}\left\|\sum_{n \in S} e^{2 \pi i n x}\right\|_{2}^{2}=a_{0} \cdot \#(S) .
$$

Proof. Suppose the theorem is false. Following the lines of the proof of Theorem 6 we could find a sequence of intervals $\left\{I_{i}\right\}_{i=1,2, \ldots}$ such that for every finite sum of exponentials $\sum$,

$$
\int_{I_{1}}\left|\sum\right|^{2} \leqslant\left(2^{-i-1}\right)^{2}\left\|\sum\right\|_{2}^{2}, \quad i=1,2, \ldots
$$


Let $K_{i}(x)=i$ on $I_{i}$ and 0 elsewhere on $[0,1]$ and let $K(x)=\sum K_{i}(x)$. Then if $f$ is the characteristic function of the set $\left\{n_{1}, \ldots, n_{N}\right\} \subset \mathbf{Z}$, so that $\hat{f}=\sum e^{2 \pi i n_{1} x}$, then the mapping

$$
T: f \rightarrow\left(K(x) \cdot \sum e^{2 \pi i n, x}\right)^{2}
$$

would be of restricted type $(2,2)$ on $\mathbf{Z}$ since by Plancherel's equality $\|T f\|_{2}=$ $\left\|\sum K_{i} \hat{f}\right\|_{2}$ which by Minkowski's inequality is dominated by

$$
\sum\left\|K_{i} \hat{f}\right\|_{2}=\sum\left(i^{2} \int_{I_{i}}|\hat{f}|^{2}\right)^{1 / 2} \leqslant \sum i\left(2^{-i-1}\|f\|_{2}\right)=\|f\|_{2}
$$

where (4.2) justifies the last inequality. It would then follow from Michael Cowling's generalization of Theorem 1 to amenable groups in general (see [2]), and $\mathbf{Z}$ in particular, that $T$ could be extended to a bounded operator on $l^{2}=L^{2}(\mathbf{Z})$, which would contradict the unboundedness of the multiplier $K(x)$.

Questions. We restrict ourselves to linear operators commuting with translation.

1. One outstanding question is whether for $\mathbf{T}$ or $\mathbf{R}$, very weak restricted $(2,2)$ operators are bounded on $L^{2}$. An affirmative answer would nicely generalize and unify Theorem 1 (see Figure 2).

2. The nonconstructive method of proving Theorem 7 concerning sums of exponentials yields no estimate of the value of the constant $a_{0}$. A direct proof is (at the least) tedious. ${ }^{2}$ Theorem 7 appears to be some sort of deep analytic number theory fact. The challenge is to find some number theoretical use for this theorem.

3. In the extension of the original operator to an $L^{2}$ to $L^{2}$ map, the original operator norm is shown to be stretched by no more than $\pi / \sqrt{2}$ in Theorems 2 and 3 , and 8 in Theorems 4 and 5 . (In our proof of Theorem 5, extending the operator to an $L(2,1)$ to $L(2, \infty)$ map multiplies the norm by 8 and the conclusion to the proof multiplies that norm by an additional $\Gamma\left(\frac{1}{4}\right)^{2} / \sqrt{8 \pi} \doteq 1.311$ ). (However, in Cowling's proof the original stretch of 8 is not compounded any further.) These constants all arise naturally in the proofs given and are the best one can get in those contexts. However, I do not know if any of them are sharp.

4. As can be seen from the proofs of Theorems 1 to 5 , the most straightforward theorem is Theorem 4-that w.r. $(p, p)$ and $s .(p, p)$ coincide when the underlying group is $\mathbf{T}$ and $p=2$. We do not even have $w .(p, p)$ implies $s .(p, p)$ if $p<2$ (see Zafran [8]). By duality, we cannot have $r .(p, p)$ imply $s .(p, p)$ if $p>2$. Thus $w . r$. implies $s$. is peculiar to $p=2$. There remains one (hard!) question. For linear operators commuting with translation, does $w .(p, p)$ imply $s .(p, p)$ when $p>2$ ?

\footnotetext{
${ }^{2}$ Added in proof (April 18, 1983). We now know that the supremum of the set of $a_{0}$ for which Theorem 7 holds is exactly $\max \sin ^{2} x /(\pi x)=.2306 \ldots$ See Inégalites sur des sommes $d$ 'exponentielles, by $\mathrm{J}$. Marshall Ash, Roger Jones et Bahman Saffari; and Estimations locales de sommes d'exponentielles, by $\mathrm{M}$. Déchamps, F. Piquard et H. Queffélec. These papers will appear contiguously in Comptes Rendus Acad. Sci. Paris.
} 


\section{REFERENCES}

1. P. Ash, J. M. Ash and R. D. Ogden, A characterization of isometries, J. Math. Anal. Appl. 60 (1977), 417-428.

2. M. Cowling, Some applications of Grothendieck's theory of topological tensor products in harmonic analy'sis, Math. Ann. 232 (1978), 273-285.

3. R. A. Hunt, On $L(p, q)$ spaces, Enseign. Math. 12 (1966), 249-275.

4. K. de Leeuw, On $L^{p}$-multipliers, Ann. of Math. 81 (1965), 364-379.

5. G. G. Lorentz, Some new functional spaces, Ann. of Math. 51 (1950), 37-55.

6. E. M. Stein and G. Weiss, Introduction to Fourier analysis on Euclidean spaces, Princeton Univ. Press, Princeton, N. J., 1971.

7. An extension of a theorem of Marcinkiewicz and some of its applications, Indiana Univ. Math. J. 8 (1959), 263-284.

8. M. Zafran, Multiplier transformations of weak type, Ann. of Math. 101 (1975), 34-44.

9. A. Zygmund, Trigonometric series, vol. I, (2nd rev. ed.), Cambridge Univ. Press, New York, 1968.

Department of Mathematical Sciences, DePaul University, 2323 North Seminary Avenue, Chicago, Illinois 60614 